\title{
Status of the LILAC Experiment
}

\author{
N. Saleh ${ }^{\dagger}$, P. $\operatorname{Han}^{\dagger}$, C. Keppel,* P. Gueye, ${ }^{*}$ V. Yanovsky ${ }^{\dagger}$ and \\ D. Umstadter ${ }^{\dagger}$ \\ ${ }^{\dagger}$ Center for Ultrafast Optical Science \\ University of Michigan, Ann Arbor, MI 48109, USA \\ *Dept. of Physics, Hampton University, Hampton, VA 23668
}

\begin{abstract}
We present the status of the LILAC experiment [1], including results on the propagation of 30-fs duration laser pulses in plasmas of the requisite density, and measurements of the dark current [2]. We also discuss the status of a laser upgrade, an electron beam line and plans for the future.
\end{abstract}

\section{INTRODUCTION}

The concept of optical injection of electrons in laser-produced plasma waves has generated much recent interest. We have proposed an acceleration schemes based on this concept [1], and embarked upon implementing it at the University of Michigan's Center for Ultrafast Optical Science. Variants of this concept were also proposed [3]. We have explored the parameter space necessary to implement this concept and are in the process of building the requisite laser system and diagnostics.

\section{Description of the Laser System}

The technology developed at our Center, along with the specific laser system we used, is described, respectively, in [4][5]. Fig. 1 and Fig. 2 depict a schematic of the laser system and experimental chambers. 


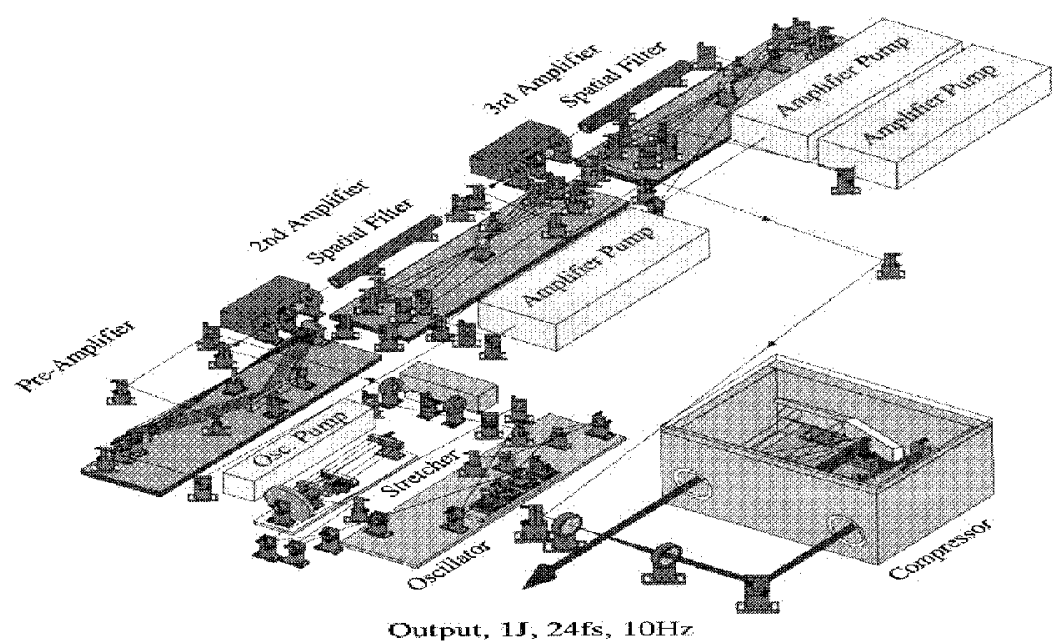

FIGURE 1. High peak power CPA laser system used in first run of the LILAC Experiment.

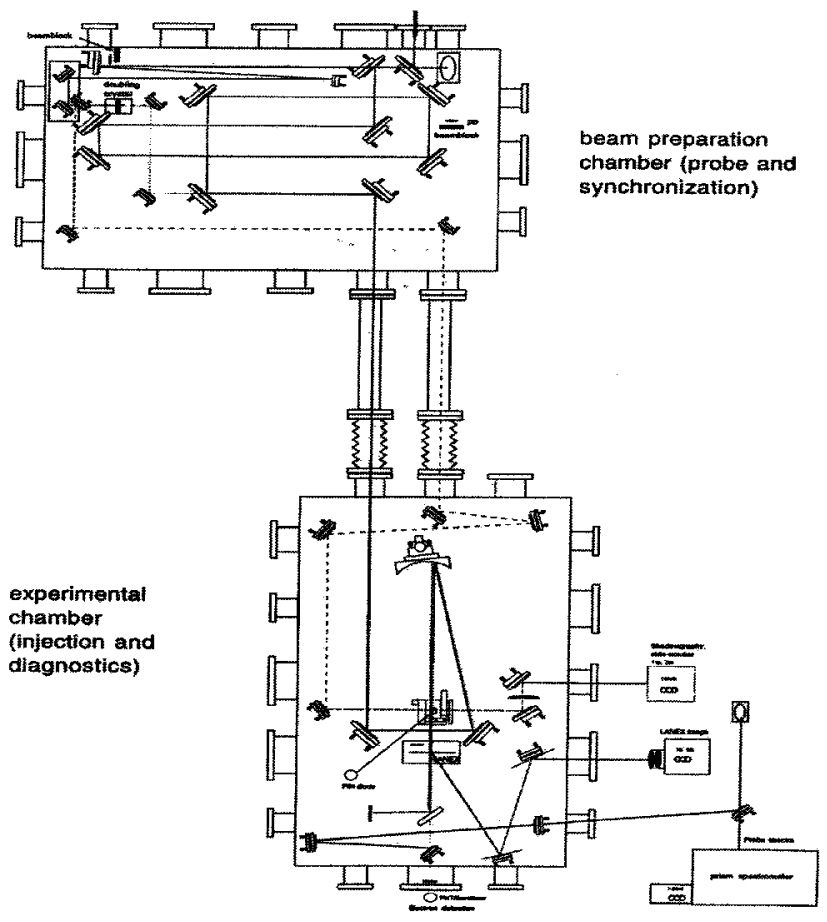

FIGURE 2. Experimental chambers for the LILAC experiment first run. 


\section{Experimental Procedure}

The LILAC (Laser Injected Laser ACcelerator) concept utilizes a loosely focused (1D) short laser pulse, which resonantly produces a wake field in under-dense plasma, and forms the acceleration structure. Another short pulse, transversely overlapped, tightly focused, and properly synchronized with the first pulse, suitably injects electrons into this acceleration structure so that they get trapped and accelerated. The resulting accelerated bunch is predicted to have novel characteristics [1]. Some issues within the experimental dynamics may cause fatally serious problems to the LILAC schemes; among these issues are, filamentation and laser beam break up, strong dark current from the resonant wakefield itself, and the failure of the short pulse to self focus in the resonant regime. We found out in our first run of the experiment that the former two points are not going to fail the LILAC scheme. The last point, however, remains to be investigated in the second run of the experiment. The experimental parameters are listed in Table1. We proceeded along with our experiment and scanned the density of the plasma by changing the backing pressure on the gas. By doing so, we were actually varying the plasma wave (wakefield) period and sweeping from the sub-resonant regime, passing through the resonant regime, and inching along towards the self-modulated regime. We had initial evidence that we were actually generating a resonant wakefiled (plasma wave) from our weak frequency-doubled probe that was collected in the forward direction from the collective collinear Thompson scattering of the anti-Stokes satellites, this is depicted in Fig. 3. We were interested in experimental observations near the resonant regime; the favorable regime for the LLAC scheme. The resonant density, $\mathrm{n}_{r}$, is related to the critical density, $\mathrm{n}_{c}$, of the plasma by the relation

$$
\mathrm{n}_{r}=\left(\lambda_{\text {laser }} / \mathrm{c} \tau_{\text {laser }}\right)^{2} \mathrm{n}_{c}
$$

with $\lambda_{\text {laser }}$ being the laser wavelength, and $\tau_{\text {laser }}$ being the laser pulse duration. Fig. 4 , shows that the laser pulse can be sustained before, and suitably after the resonant regime without breaking up. This range extends beyond the critical power for relativistic self-focusing, and up to five times the critical power where relativistic filamentation takes place. Only then, we observe the generation of narrow (1-degree)

\section{TABLE 1. Experimental parameters of the first run of LILAC experiment}

\begin{tabular}{|l|l|}
\hline Laser pulse duration & $29 \mathrm{fs}$ \\
\hline Laser wavelength & $810 \mathrm{~nm}$ \\
\hline Maximum power & $35 \mathrm{TW}$ \\
\hline Maximum intensity & $3 \times 1018 \mathrm{~W} / \mathrm{cm}^{2}$ \\
\hline Plasma medium & He-like $\mathrm{N}_{2} @ n_{e} \leq 10^{20} \mathrm{~cm}^{-3}$, supersonic gas jet \\
\hline Laser beam diameter & $4 \mathrm{~cm}$ \\
\hline Focusing parabola & $\mathrm{f} / 4.5(130)$. \\
\hline
\end{tabular}


MeV-electron bursts correlated with emission of red-shifted laser light, unlikely from forward Raman scattering [6]. We also show in [2] how the filaments' characteristics closely compare to what was theoretically predicted.

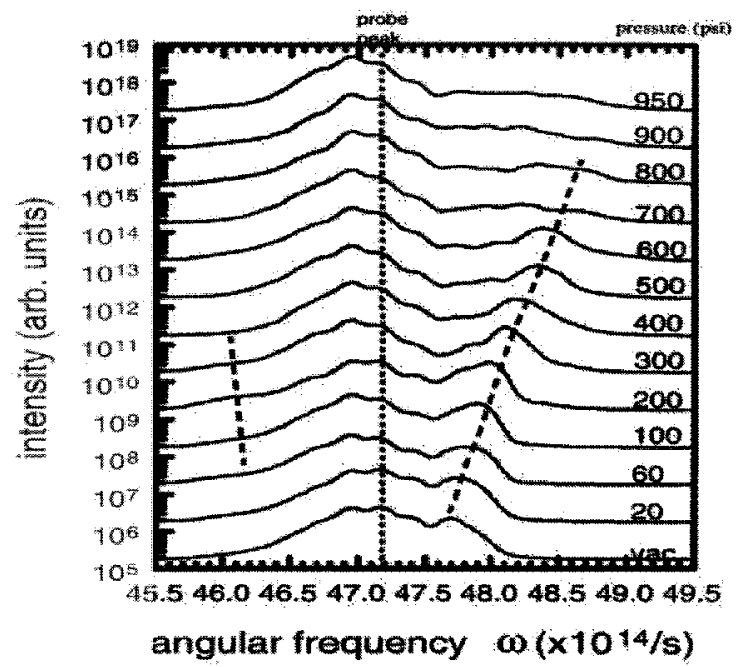

FIGURE 3. Initial evidence of collective collinear Thompson scattering of the anti-Stoles satellites from a resonant wakefield; satellite position varies with gas backing pressure (plasma period).

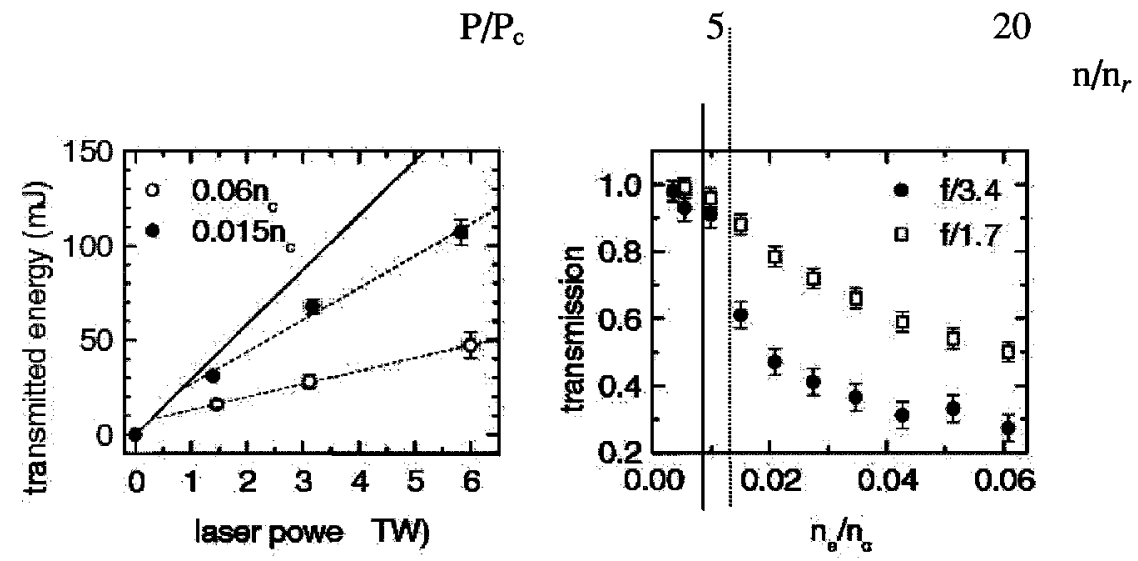

FIGURE 4. Data collected from the transmitted laser light when focused onto the supersonic gas jet. When using the $f / 3.4$ collecting optic, and at five times the critical power, we observe strong filamentation, and the diffracted light exiting the gas jet significantly misses the collecting optic. The solid line represents the resonant regime, when both ratios; $\mathrm{n} / \mathrm{n}_{r}$ and $\tau_{\text {laser }} / \tau_{p l}$ are unity $\left(\tau_{p l}\right.$ is the plasma wave duration). The dotted line represents the onset of relativistic filamentation, and MeVelectron generation.

We emphasize here that electrons were observed only when filamentation took place, but prior to that, including the vicinity of the resonant regime, no significant electron 
signal was measured. This brings us to the conclusion that there was no dark current in the resonant regime, and that the LILAC operational power parameters are:

$P_{C}<P<5 P_{C}$, with $P_{C}$ being the critical power for relativistic self-focusing. This concludes the data set for the first experimental run. The significance of the absence of dark current, i.e., pre-injection self-trapped electrons, is that the resonant wakefield will not be distorted or beam-loaded, so that the predicted novel characteristics (monochromaticity, duration, contrast, transverse emittance, etc.) of the accelerated bunch, after injection, will not be compromised.

\section{Laser System Upgrade}

We are developing and new laser system at the Center for Ultrafast Optical Science of the University of Michigan [8]. The new system is a high contrast, CPA, 150 TW on-target, with a dual compressor system, which permits the independent control of the optical properties of two laser pulses to optimize them for production of the wakefiled, and injecting electrons in it. A simple schematic is shown below. We anticipate the system to be completed no later than the end of the current year.

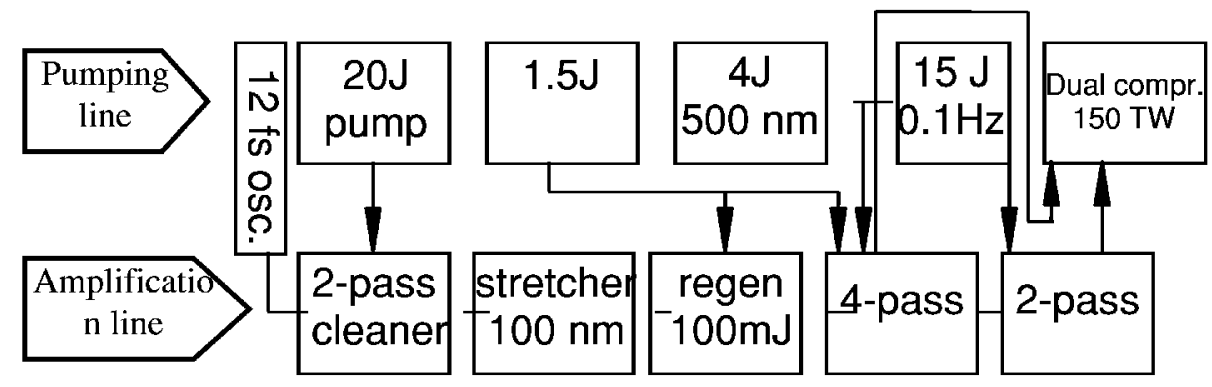

\section{QQQDD Electron Spectrometer System}

We have also developed a $500-\mathrm{MeV}$ electron spectrometer at the University of Michigan's Center for Ultrafast Optical Science with collaborators from Hampton University [7]. The QQQD part of the spectrometer has been installed in the experimental area at Michigan, and ready to receive beam. We await the last dipole to arrive at some later time. This system was designed and simulated using two beam transport codes; TRANSPORT and OPTIM. Another code, GEANT, was also used to investigate the generation of secondary particles from the beam pipe. Fig. 5 to Fig. 8 show some details of this spectrometer design. The magnets were manufactured by Danfysik, and tested, calibrated, and characterized by Hampton University using a rotating coil probe with $\% 0.1$ and $\% 1$ accuracy for the dipoles and quadrupoles, respectively. 


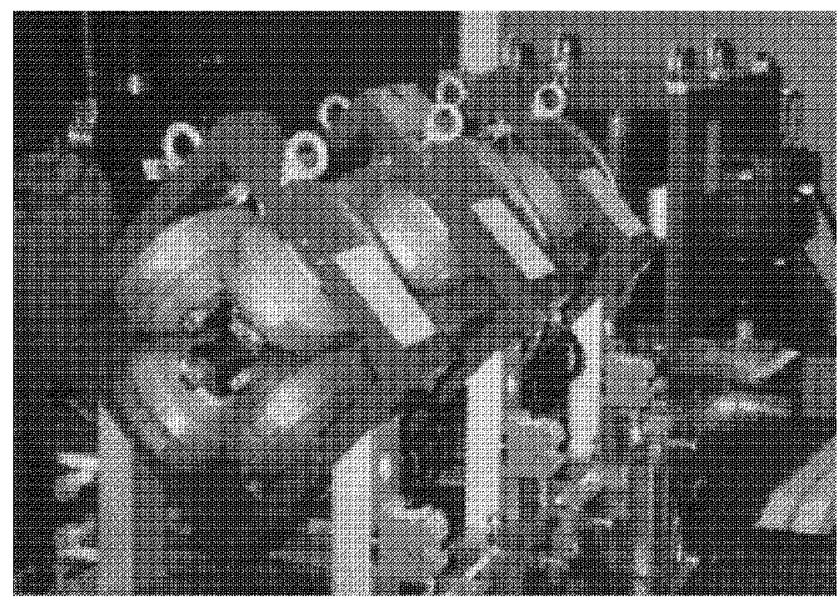

FIGURE 5. Spectrometer under construction, three quadrupoles, and one dipole.

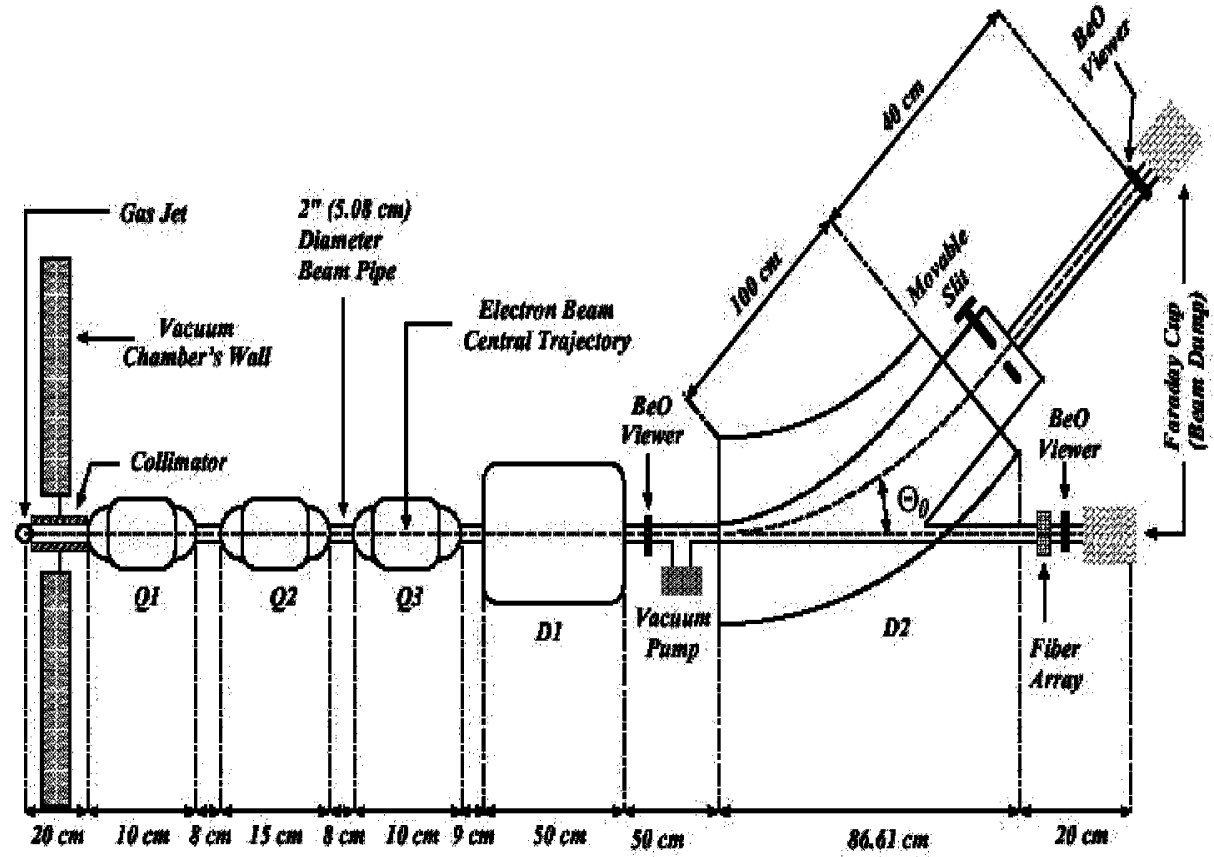

FIGURE 6. Schematic of the electron spectrometer at CUOS, Michigan. 


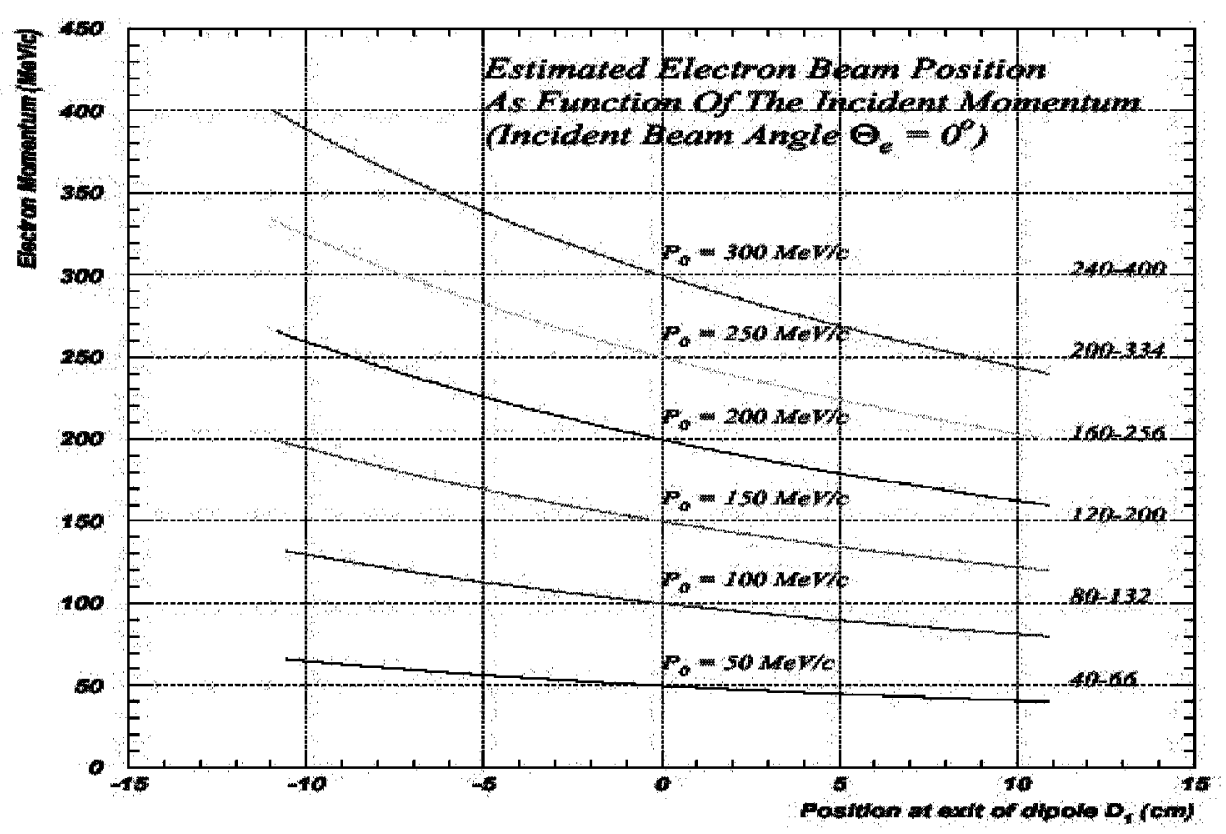

FIGURE 7. Dispersion curves generated by OPTIM at the exit of the first dipole magnet for various electron momenta.
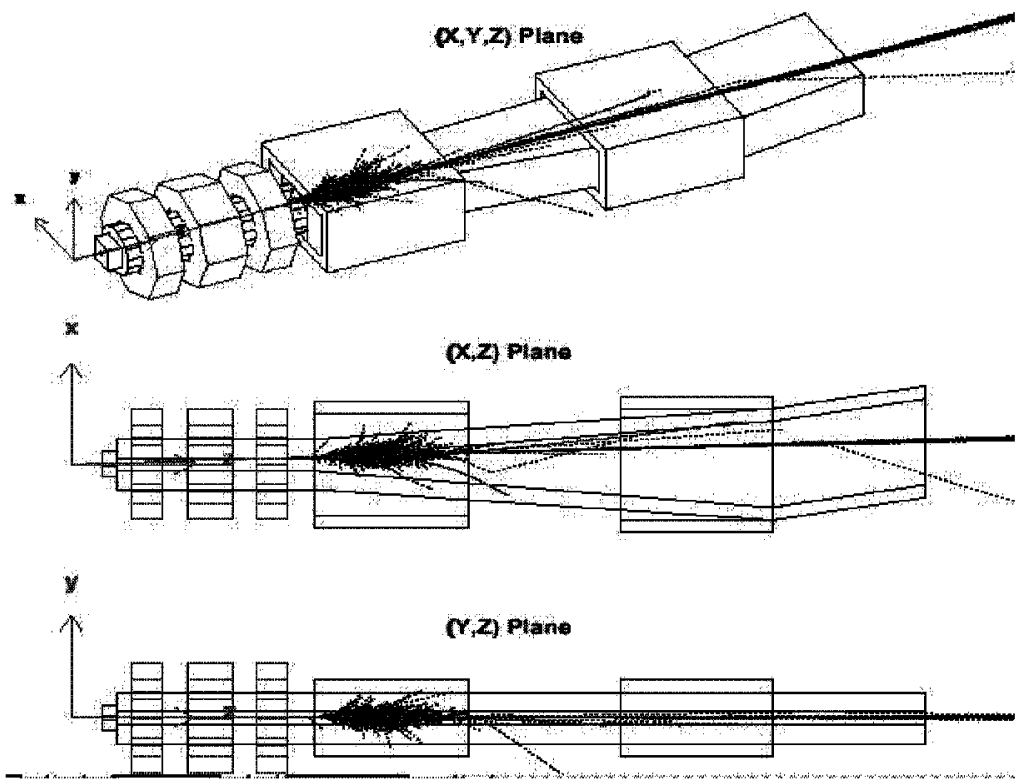

FIGURE 8. GEANT simulation of secondary particles production for a worst-case-scenario of 50 $\mathrm{GeV}$ incident electron beam. 


\section{In-Chamber Coherent Transition Radiation (CTR) Detector}

We also plan on installing a coherent transition radiation detector inside the vacuum chamber where the experiment optics are set up. The reason for doing so is that the LILAC scheme, predicts the productions of an electron bunch as short as $10 \mathrm{fs}$, this corresponds to infrared CTR of 3 microns and longer, and we are not aware of any window material with transmission properties that cover such a broad band of radiation. The setup for this detector consists of a generic Michelson interferometer, with a broadband pyroelectric IR detector from Molectron. See Fig. 9.

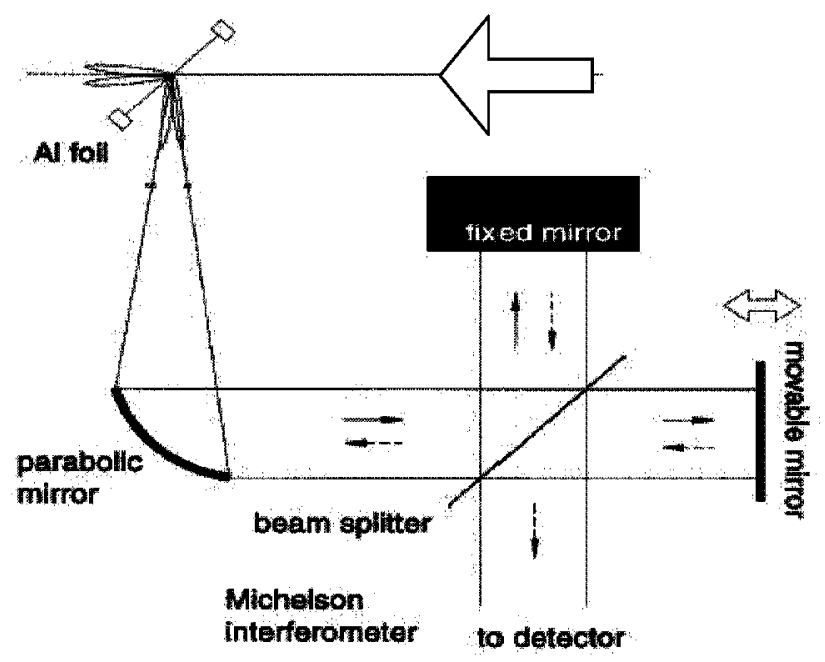

FIGURE 9. Schematic for CTR detector intended for the second run of the LILAC experiment.

\section{CONCLUSION}

The first run of the LILAC experiment produced forthcoming results in terms of the feasibility of the LILAC scheme. The resonant-wake-producing laser pulse was sustained in the plasma above the critical power for relativistic self-focusing, and the produced wake did not self-trap and accelerate any significant charge (no dark current). The Laser system is being upgraded to operational specifications closer to the optimum experimental conditions than before. We also designed an electron spectrometer that will be used to analyze the produced electron bunch. Other diagnostics are also being developed to provide full characterization of the anticipated accelerated bunch. This promotes the LILAC scheme to a promising run in the near future. 


\section{ACKNOWLEDGEMENT}

We acknowledge the support of the High Energy Physics Division of the US Department of Energy, with laser facilities supported by the NSF. We would also like to thank X. Wang, G. Mourou, H. Kapteyn, M. Murnane and S. Backus for their many contributions.

\section{REFERENCES}

1. D. Umstadter et al., Physical Review Letters, 76, 2073 (1996).

2. X. Wang et al, Physical Review Letters, 84, 5324 (2000).

3. E. Esarey et al., Physical Review Letters, 79, 2682 (1997), B. Rau et al., Physical Review Letter, 78, 3310 (1997), R. G. Hemker et al., Phys. Rev. E, 57, 5920 (1998), S. Bulanov, Plasma Phys. Rep. 25, 468 (1999), C.I. Moore et al., Physical Review Letters. 82, 1688 (1999).

4. S. Backus et al., Review of Scientific Instruments, 69, 1207 (1998).

5. H. Wang et al., JOSA B, 16, 1790 (1999).

6. See details in reference [1] on this point.

7. P. Gueye et al, proposal to be published.

8. V. Yanovsky et al., Digest of CLEO Technical Papers 2000, 288 (2000). 